\title{
A Novel Vaccine Delivery Model of the Apicomplexan Eimeria tenella Expressing Eimeria maxima Antigen Protects Chickens against Infection of the Two Parasites
}

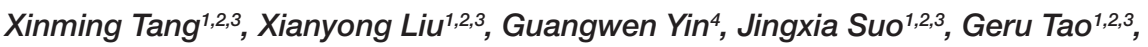 \\ Sixin Zhang ${ }^{1,2,3}$ and Xun Suo ${ }^{1,2,3 *}$ \\ 'State Key Laboratory of Agrobiotechnology, College of Veterinary Medicine, China Agricultural University, Beijing, China, \\ ${ }^{2}$ Key Laboratory of Animal Epidemiology of the Ministry of Agriculture, College of Veterinary Medicine, China Agricultural \\ University, Beijing, China, ${ }^{3}$ National Animal Protozoa Laboratory, College of Veterinary Medicine, China Agricultural University, \\ Beijing, China, ${ }^{4}$ Engineering Laboratory of Animal Pharmaceuticals, College of Animal Science, Fujian Agriculture and \\ Forestry University, Fuzhou, Fujian, China
}

OPEN ACCESS

Edited by:

José Roberto Mineo, Federal University of Uberlandia,

Brazil

Reviewed by:

Herbert Leonel de Matos Guedes, Universidade Federal do Rio de

Janeiro, Brazil

Michael Kogut,

Agricultural Research Service (USDA), United States

*Correspondence: Xun Suo

suoxun@cau.edu.cn

Specialty section:

This article was submitted to

Microbial Immunology,

a section of the journal

Frontiers in Immunology

Received: 25 July 2017 Accepted: 20 December 2017 Published: 10 January 2018

Citation:

Tang X, Liu X, Yin G, Suo J, Tao G, Zhang $S$ and Suo $X$ (2018) A Novel

Vaccine Delivery Model of the Apicomplexan Eimeria tenella

Expressing Eimeria maxima Antigen Protects Chickens against Infection of the Two Parasites.

Front. Immunol. 8:1982. doi: 10.3389/fimmu.2017.01982
Vaccine delivery is critical in antigen discovery and vaccine efficacy and safety. The diversity of infectious diseases in humans and livestock has required the development of varied delivery vehicles to target different pathogens. In livestock animals, previous strategies for the development of coccidiosis vaccines have encountered several hurdles, limiting the development of multiple species vaccine formulations. Here, we describe a novel vaccine delivery system using transgenic Eimeria tenella expressing immunodominant antigens of Eimeria maxima. In this delivery system, the immune mapped protein 1 of E. maxima (EmIMP1) was delivered by the closely related species of E. tenella to the host immune system during the whole endogenous life cycle. The overexpression of the exogenous antigen did not interfere with the reproduction and immunogenicity of transgenic Eimeria. After immunization with the transgenic parasite, we detected EmIMP1's and E. maxima oocyst antigens' specific humoral and cellular immune responses. In particular, we observed partial protection of chickens immunized with transgenic E. tenella against subsequent E. maxima infections. Our results demonstrate that the transgenic Eimeria parasite is an ideal coccidia antigen delivery vehicle and represents a new type of coccidiosis vaccines. In addition, this model could potentially be used in the development of malaria live sporozoite vaccines, in which antigens from different strains can be expressed in the vaccine strain.

Keywords: vaccine delivery model, transgenic Eimeria, immune mapped protein 1, protective immunity, Eimeria maxima

\section{INTRODUCTION}

Apicomplexan genera parasites that cause serious human, veterinary, or zoonotic diseases include Plasmodium, Toxoplasma, Eimeria, Neospora, and Theileria (1-4). Chicken coccidiosis caused by the genus Eimeria parasites occurs in almost all the poultry farms, results in approximately $£ 2$ billion in losses per year and limits the development of the modern poultry industry (5). It has been proposed that the modern poultry industry could have never developed to its present extent without 
the advent of drugs to control coccidiosis (6). Most drugs are no longer as effective as when they were first introduced due to the development of drug resistance. There is also risk of potential drug residues in poultry meat that threaten food security. Both problems constitute major limitations of anti-coccidiosis drugs $(6,7)$. Instead of drugs, vaccination with formulations containing either the virulent or the attenuated live parasites has been considered the most efficient method for the protection of breeder and layer flocks from Eimeria spp. infection, preventing the presence of drug residues in poultry productions $(8,9)$. Vaccinated chicks with one Eimeria species showed complete defense against subsequent homogeneous infection after self-boosting, but not against heterogeneous parasites $(7,9,10)$. These vaccine formulations with Eimeria parasites have resulted in commercially available anticoccidial vaccines with live parasites formulations containing multiple species of Eimeria. Some require at least three Eimeria species (Eimeria acervulina, Eimeria maxima, and Eimeria tenella) for broilers and five for layers and breeders. However, the requirement of having to simultaneously maintain multiple parasite lines and their production capacity, in addition to economics costs, have been proven to be hurdles to advance the development of these multiple species vaccine formulations $(5,11)$. Reduction of live parasites formulations, especially of the pathogenic species of the anti-coccidiosis vaccines, can be beneficial in improving animals' welfare and reducing vaccine production cost.

Eimeria maxima is the most immunogenic among the seven species of the Eimeria parasites that infect chickens, causing pathological damage in the mild intestine. Infection with as few as five E. maxima oocysts can induce complete protective immunity against subsequent homologous challenges $(5,10)$. The degree of cross-protection among different strains of E. maxima is quite variable. In contrast, all six other species of Eimeria are perfectly protected against different strains of the same species. Previous studies have shown that just six regions in the E. maxima genome were targeted by immunity and two of these have now been interrogated to determine the protective antigen-encoding gene. A new highly protective antigen for E. maxima, termed immune mapped protein 1 (IMP1), was identified using genetic mapping techniques (12). Vaccination with the recombinant IMP1 of E. maxima by intramuscular injection induces an immune response but provides only partial protection. In addition, none of the vaccine candidates has been tested in commercial applications $(5,11)$. However, since protective antigens may not be identified until a suitable method of delivery has been developed and a suitable method of delivery may not be recognized until protective antigens have been isolated, the present scenario for the identification of putative immunogenic molecules of Eimeria represents a classic "Catch 22 " situation $(5,11)$. We hypothesized that an antigen delivery vehicle carrying protective antigens to target the intestinal mucosal immune system would be the best approach for the development of recombinant anti-coccidiosis vaccines based on one or several immune protective antigens.

Eimeria tenella, the model organism to study the biological and immunological characteristics of the apicomplexan parasite, has been considered as vaccine delivery vehicle expressing pathogen antigens. These elicit foreign antigen-specific immune responses in chickens and in mammals (13-15). Since there are another six species of Eimeria parasites that infect chicken intestinal mucosa in addition to E. tenella, we further hypothesized that $E$. tenella would be the best vaccine delivery vehicle to carry Eimeria parasite immunodominant antigens to target the intestinal mucosal immune system to provide protective immunity against subsequent parasites infection. To prove this idea, in this study, we constructed a transgenic E. tenella line expressing the immunodominant antigen of E. maxima (Et-EmIMP1) and used it as anticoccidial vaccine formulation to investigate its protective immunity against heterogeneous E. maxima infection in chickens.

\section{MATERIALS AND METHODS}

\section{Ethics Statement}

All animal experiments were performed in strict accordance with the China Agricultural University Institutional Animal Care and Use Committee guidelines (CAU20160628-2) and followed the International Guiding Principles for Biomedical Research Involving Animals. Experiments were approved by the Beijing Administration Committee of Laboratory Animals.

\section{Parasites and Animals}

Eimeria tenella (XJ strain), E. maxima (BJ strain), Eimeria mitis (CAU strain), and E. acervulina (CAU strain) used in this study were maintained by propagating them in coccidian-free, 2 - to 5-week-old Arber Acres broilers. The procedures for collection, purification, and sporulation were carried out as previously described (16).

One-week-old SPF chickens were purchased from Merial Animal Health Co., Ltd. (Beijing, China) and were fed a pathogenfree diet and water ad libitum.

\section{Plasmid Construction}

Total RNA was isolated from E. maxima sporozoites was isolated using the TRIzol reagent (Invitrogen, USA).cDNA was synthesized through the utilization of random primers and a High Capacity cDNA Reverse Transcription Kit (Applied Biosystems, USA). The open reading frame of E. maxima IMP1 was amplified by PCR using IMP1-F/IMP1-R1 primers (Table S1 in Supplementary Material) designed according to the IMP1 sequences (GeneBank Accession number: FN813228.1). Flag-tag was targeted at the 3 ' end of IMP1 gene by two rounds overlapping PCR using IMP1-F/IMP1-R2 and IMP1-F/IMP1-R3, respectively (Table S1 in Supplementary Material). After PCR amplification, Nde I and Sac II restriction enzyme sites were introduced into IMP1 fragment. The transfection vector, pSDEP2AIMP1S, was constructed based on pSDEP2ARS (17). Briefly, the ssRFP-His tag fragment of pSDEP2ARS was replaced by IMP1-Flag-tag fragment based on Nde I and Sac II restriction enzymes digestion and DNA ligase ligation. The transfection vector was linearized by SnaB I restriction enzyme for further use.

\section{Construction of the Transgenic E. tenella Line}

A restriction enzyme-mediated integration was adapted for the transfection of E. tenella sporozoites as previously described 
(18). Stably transfected E. tenella line was obtained by pyrimethamine selection combined with fluorescence activated cell sorting (Table S2 in Supplementary Material). Western blot and indirect immunofluorescent assay (IFA) were conducted to confirm foreign antigens expression and distribution in transgenic parasites based on the previous described protocols, respectively (15). Sporozoites were extracted from fresh collected sporulated oocysts. First and secondary schizonts and gametocytes were collected from variable dose EmIMP1 $\left(5 \times 10^{7}, 1 \times 10^{7}\right.$, and $1 \times 10^{3}$ oocysts, respectively) infected chickens at 3,5, and $7 \mathrm{dpi}$ using the previous methods (19). Soluble antigens of sporozoites, first-generation schizonts, second-generation schizonts, gametocytes, and unsporulated oocysts were resolved by SDS-PAGE and immunoblot analysis following standard protocols with mouse anti-flag monoclonal antibody and HRP-conjugated goat anti-mouse IgG (Proteintech, USA) as primary and secondary antibody, respectively. Poly-antibodies against E. tenella GAPDH and enhanced yellow fluorescent protein (EYFP) served as controls.

Indirect immunofluorescent assays were conducted to detect the distribution and relative expression level of foreign antigen. The primary and secondary antibody were mouse anti-flag monoclonal antibody and Cy3-conjugated goat anti-mouse IgG (Proteintech, USA). Poly-antibodies against endogenous IMP1 of E. tenella served as control.

\section{Quantification of Transgenic Parasite Replication}

Three groups of four inbred SPF chickens were inoculated with 100 sporulated wild-type E. tenella, EtER (transgene control) and Et-EmIMP1 oocysts at 1 week of age. The output of oocysts of the transgenic lines and wild-type parasite in chickens was measured daily by using McMaster egg counting chamber between 4 and 14 days' postinoculation, respectively (20).

\section{Observation of EYFP Expression in the Endogenous Development of the Transgenic Parasites}

Six groups of two inbred SPF chickens were inoculated with $5 \times 10^{7}, 1 \times 10^{7}, 1 \times 10^{6}, 1 \times 10^{5}, 1 \times 10^{4}$, and $1 \times 10^{3}$ sporulated Et-EmIMP1 oocysts at 1 week of age. Chickens were restricted to feed to reduce the amount of cecum. For each infection dose groups, chickens were euthanized every $24 \mathrm{~h}$ for necropsy. The cecum tissue was washed three times using cold PBS. Fresh smears were made from pieces of tissues from the mucous membrane of the cecum and visualized using a confocal laser scanning microscope (SP5, Leica, Germany) for the detection of EYFP-expressing parasites.

\section{Vaccination and Challenge Infection}

Four groups of six inbred SPF chickens were either left naïve or were immunized by infection with 200 sporulated wild-type E. tenella, EtER, and Et-EmIMP1 oocysts at 1 week of age. Secondary immunization was administrated at 2-week intervals with 1,000 oocysts as immunization dosage for each bird. All chickens were housed in the same conditions of temperature and humidity and fed a coccidian-free diet and water ad libitum. Serum was collected when chickens were 1,3 , and 5 weeks old and stored at $-20^{\circ} \mathrm{C}$ until use. Challenge infections with wildtype E. tenella (50,000 oocysts) or E. maxima (50 oocysts) were conducted 2 weeks after the last immunization (at 5 weeks of the chicken age). Oocyst output and body weight gain of the chickens were evaluated after challenge infection.

\section{ELISA and ELISPOT}

ELISA was conducted as previously described $(15,21)$. Briefly, $5 \mu \mathrm{g} / \mathrm{ml} \mathrm{E}$. tenella or E. maxima oocysts antigens or $1 \mu \mathrm{g} / \mathrm{ml}$ recombinant EmIMP1 were coated onto the individual wells of the plates followed by a reaction with serum $(1: 100)$ and collected when chicken were 1,3 , and 5 weeks old. The secondary antibody used in this experiment was the HRP-conjugated goat anti-chicken IgY Fc fragment (Bethyl Laboratories, Inc.). Foreign antigen and parasites specific cellular immune responses revealed by IFN- $\gamma$ secreting cells present in peripheral blood mononuclear cells (PBMCs) after immunization with the transgenic parasite or its wild-type parasite were evaluated by ELISPOT following established protocols in our laboratory (22). Briefly, $10^{6} \mathrm{PBMCs}$ from the naïve, the wild-type E. tenella, EtER, and the Et-EmIMP1 oocysts immunized birds were stimulated with $10 \mu \mathrm{PBS}, 10 \mu \mathrm{g}$ E. tenella or E. maxima oocysts antigen or $2 \mu \mathrm{g}$ recombinant EmIMP1, and $10 \mu \mathrm{l} \mathrm{PMA} \mathrm{plus} \mathrm{ionomycin} \mathrm{(10} \mathrm{ng/ml} \mathrm{PMA} \mathrm{plus}$ ionomycin $5 \mu \mathrm{g} / \mathrm{ml}$ ), respectively. The spots in which IFN- $\gamma$ secretion lymphocytes were present were detected after $24 \mathrm{~h}$ stimulation.

\section{Floor Pen Test}

Groups of 12 inbred SPF chickens were either left naïve (Ctrl) or were immunized by infection with 200 sporulated wild-type E. tenella (WT), EtER, and Et-EmIMP1 oocysts at 1 week of age. Chickens were housed in the same conditions of temperature and humidity. New litter of chopped straw was spread over cages' bottom $5 \mathrm{~cm}$, and chickens were fed a coccidian-free diet and water ad libitum. Six chickens of each group were removed to new cages and challenged with E. maxima (50 oocysts/bird) at 14 and $28 \mathrm{dpi}$, respectively. Oocyst outputs were evaluated after each challenge infection.

To test the species-specific protective immunity elicited by Et-EmIMP1, groups of 12 inbred SPF chickens were either left naïve (Ctrl) or were immunized by infection with 200 sporulated wild-type E. tenella (WT), Et-EmIMP1 oocysts at 1 week of age. Chickens were housed in the same conditions of temperature and humidity and fed a coccidian-free diet and water ad libitum, while all the chickens were infected with E. acervulina or E. mitis (50 oocysts/bird) at 14 and $28 \mathrm{dpi}$.

\section{Statistical Analysis}

GraphPad Prism 6.01 (GraphPad Software) was used for statistical analysis. Differences between control and treated groups were analyzed using SPSS 12.0 (SPSS Institute Inc.). Differences in the experimental treatments were tested using Duncan's Multiple Range Test following ANOVA with significance reported at $p \leq 0.05$. 


\section{RESULTS}

\section{Construction of Transgenic E. tenella Line Expressing E. maxima IMP1 (Et-EmIMP1)}

Techniques for the selection of stably transfected Eimeria parasites have been successfully developed in the last decade (23-25). To improve the transfection efficiency, here we constructed a relatively short plasmid co-expressing the reporter and target genes (immune mapped protein 1 of E. maxima, EmIMP1) mediated by $\mathrm{P} 2 \mathrm{~A}$ sequence in a single expression cassette plasmid [Figure 1A; Table S1 in Supplementary Material; (17)]. We obtained the stably transfected parasite (Et-EmIMP1) expressing the reporter gene after an exhaustive selection in vivo (Figure 1B; Table S2 in Supplementary Material) and confirmed that the exogenous plasmid was integrated into the Eth_scaff 16 locus of the Et-EmIMP1 genome (Figure 1C). We also analyzed the oocysts shedding pattern and total oocyst output of the chickens after inoculation with Et-EmIMP1 and its wild type to reveal the reproduction of the parasites affected by the transgenic manipulation. We found similar oocyst shedding patterns as well as total oocyst output for Et-EmIMP1 and its wild type (Figures 1D,E). The peak corresponding to the maximum oocyst output for the transgenic manipulation control [EtER, a transgenic E. tenella line described previously (17)] showed a 24 -h delay respect to the wild type (WT) and Et-EmIMP1 (Figure 1D). These data demonstrated that we obtained a pure transgenic Eimeria line to investigate its immunogenicity.

\section{EmIMP1 Was Highly Expressed in the Diverse Endogenous Developmental Stages}

The schizogony stages are considered to be the most immunogenic of the diverse endogenous developmental stages undergone by eimerian coccidian (10). To observe the EmIMP1 expression pattern during the complex life cycle of the transgenic parasite, we first confirmed that the co-expressing reporter gene was continuously expressed in the diverse endogenous developmental stages of Et-EmIMP1 as sporozoite, trophozoite, schizont, and gametocyte, but not in the unsporulated oocyst stage (Figure 2A). These results suggested that EmIMP1 was expressed in the whole life cycle of Et-EmIMP1, as both EYFP and EmIMP1 were controlled by one set of regulatory elements (17). To further confirm the expression pattern, we conducted an immunoblot assay. Consistent with the expression of EYFP, EmIMP1 was highly expressed in the diverse endogenous developmental stages of the transgenic parasite, except in the unsporulated oocyst stage (Figure 2B).

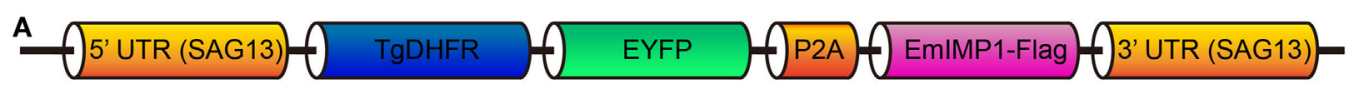

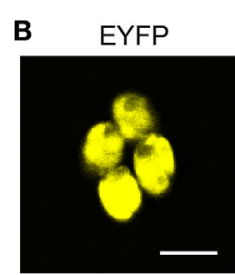
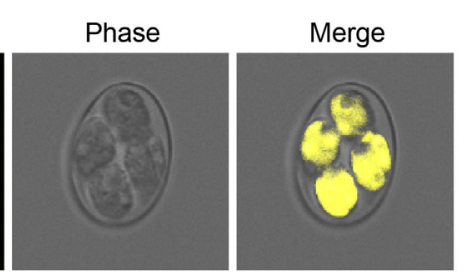

C

E. tenella chromosome

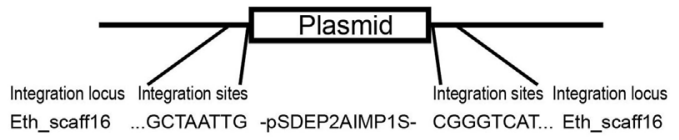

D

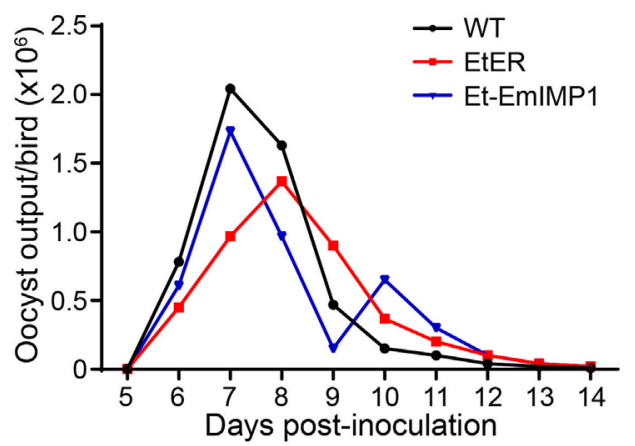

E

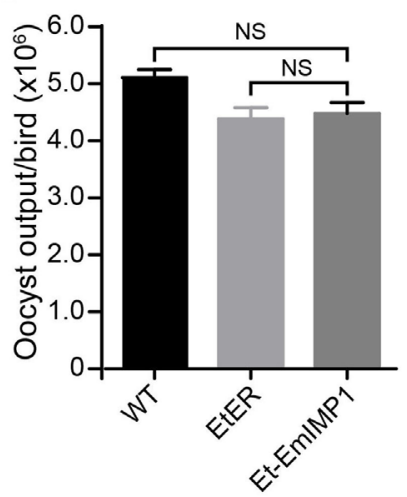

FIGURE 1 | Construction of transgenic Eimeria tenella expressing immune mapped protein 1 of E. maxima (EmIMP1) and its reproduction. (A) EmIMP1 with a flag-tag was co-expressed with TgDHFR-EYFP and linked by P2A in the single expression cassette. (B) Stably transfected EmIMP1 expressing reporter enhanced yellow fluorescent protein (EYFP) in its sporulated stage. Bar $=10 \mu \mathrm{m}$. (C) The exogenous DNA was integrated into Eth_scaff16 according to its flanking sequences obtained by genome walking technique. (D) Comparison of oocyst shedding patterns of Et-EmIMP1, its wild type (WT), and the transgenic manipulation control (EtER). The output of oocyst from the three $E$. tenella lines in the parasite infected chickens was measured daily between 5 and 14 days postinoculation $(n=3)$. (E) The reproduction of Et-EmIMP1 after inoculation was similar to its wild type and transgenic manipulation control (EtER), as measured by their total oocyst output. NS, not significant. Each value represents the mean \pm SD of three birds. 


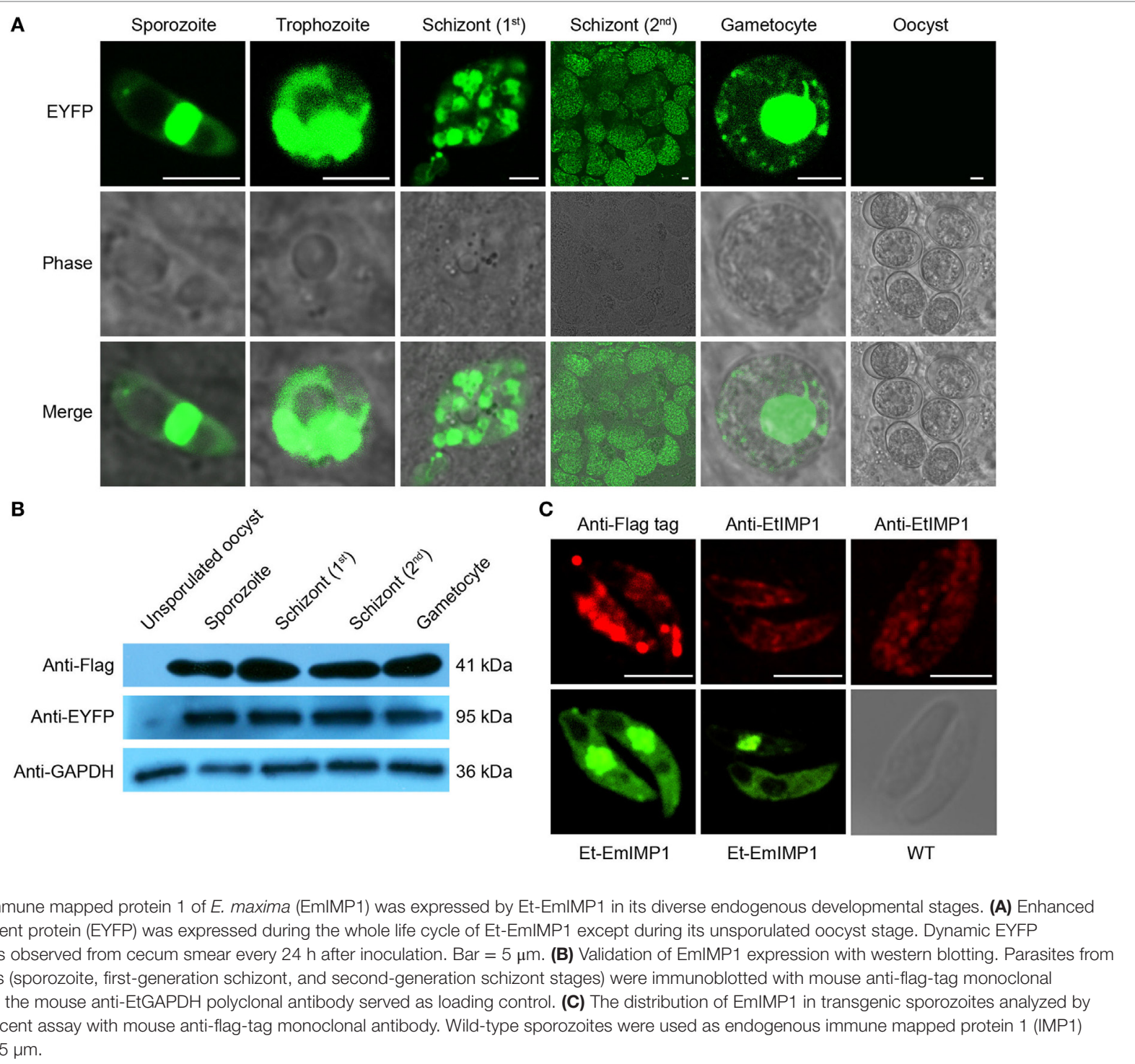

Homologs of the IMP1 gene can be readily identified in eimerian and non-eimerian apicomplexan parasites, and these may also be candidate protective antigens $(12,26,27)$. The IMP1 of E. tenella is an immunodominant antigen that elicits partial protective immunity against E. tenella infection (28). To determine whether the exogenous EmIMP1 with such high level of expression would interfere with the endogenous EtIMP1, we analyzed the expression and distribution of EmIMP1 and endogenous EtIMP1 for the transgenic parasite. We found that EmIMP1 was expressed in the whole sporozoites of Et-EmIMP1 except in the refractile body (Figure 2C). The endogenous EtIMP1 was expressed mainly on the cell surface of Et-EmIMP1 sporozoites (Figure 2C), as reported in previous experiments with transient transfection of E. tenella sporozoites with EtIMP1 tagged with red fluorescent protein (28). In general, EmIMP1 was continuously highly expressed during the whole life cycle of Et-EmIMP1 and did not interfere with the endogenous EtIMP1.

\section{Et-EmIMP1 Protects Chickens from Parental Parasite Infection}

Most Eimeria parasites are immunogenic pathogens that elicit systemic and mucous immunity against parental parasite reinfection (29). With few exceptions, transgenic manipulation of Eimeria parasites expressing molecules not relevant for the immune response did not affect their immunogenicity $(25,30)$. To evaluate the immunogenicity of our transgenic population expressing high levels of immune molecules compared with its parental strain, we first investigated the cell-mediated immune response stimulated by Et-EmIMP1. This response is revealed by the ratio of parasites' antigen-specific IFN- $\gamma$ secreting cells in PBMCs after immunization, as they play a major role in protecting the host from Eimeria parasite reinfection (31). We found no significant difference between Et-EmIMP1, its wildtype immunized birds or the transgenic control (EtER) for the E. tenella oocyst antigens (Et Ag)-specific in IFN- $\gamma$ secreting cells 
in PBMCs (Figures 3A,B). Humoral immunity also contributes to provide additional immunity against Eimeria parasites infection, especially against E. maxima infection (32). As expected for a cell-mediated immune response, we found that Et-EmIMP1 did not reduce antibody production after immunization (Figure 3C).

To assess the protective efficacy of Et-EmIMP1 as an avian coccidiosis vaccine strain against its parental parasites infection, we infected Et-EmIMP1 immunized birds by oral inoculation with wild-type E. tenella. We found there was no oocyst output neither in Et-EmIMP1 nor in its wild-type immunized birds when the challenge dose was 10,000 oocysts (data not shown). When the challenge dose was 50,000 E. tenella oocysts, the oocyst output was significantly reduced compared to naïve chickens (Figure 3D). With reduced oocyst output, both Et-EmIMP1 and wild-type parasite immunized birds gained much more body weight (Figure 3E). Taken together, these results clearly show that Et-EmIMP1 maintained the immunogenicity of its wild-type parasite and indicate that a coccidiosis vaccine formulation will protect chickens against $E$. tenella infection.

\section{Et-EmIMP1 Elicits EmIMP1-Specific Immune Responses That Protects Chickens from E. maxima Infection}

Vaccination with live parasites (attenuated or un-attenuated strains) is the most powerful way to control coccidiosis in chickens $(5,6,10)$. Commercial vaccines against coccidiosis based on live parasites contain multiple species, as there is no cross-protection stimulated by a single parasite. The results presented above showed that Et-EmIMP1 maintained its paternal strain's immunogenicity protecting chickens from E. tenella infection. As next step, we evaluated the EmIMP1 and E. maxima-specific immune responses elicited by Et-EmIMP1 as well as the protective efficacy against E. maxima infection in chickens. We found that Et-EmIMP1 elicited foreign antigenspecific cellular and humoral immune responses revealed by the number of EmIMP1-specific IFN- $\gamma$ secreting cells (Figure S1A in Supplementary Material) and antibody production (Figure S1B in Supplementary Material). Immunities against

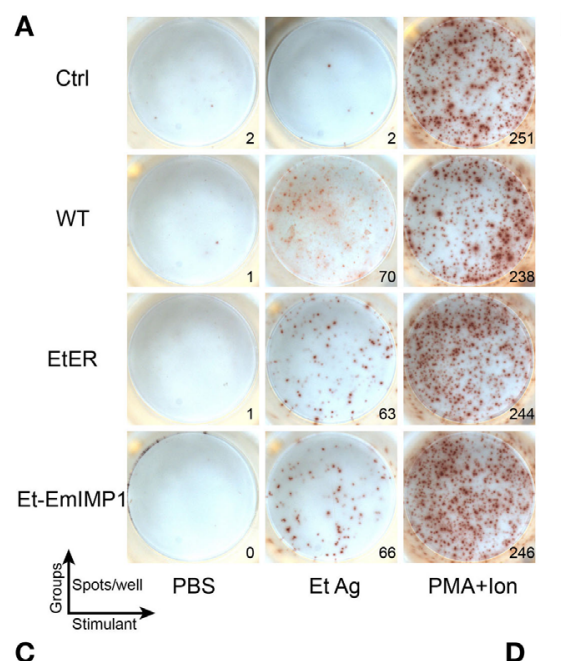

C

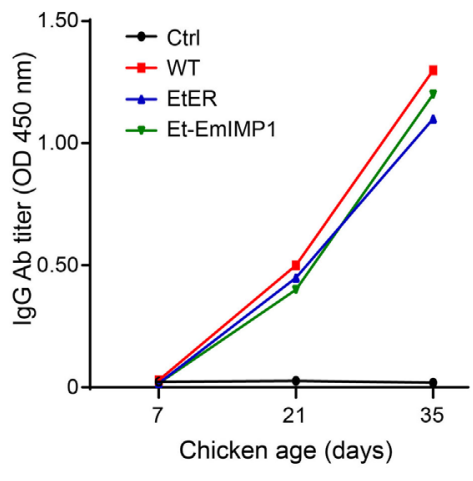

D
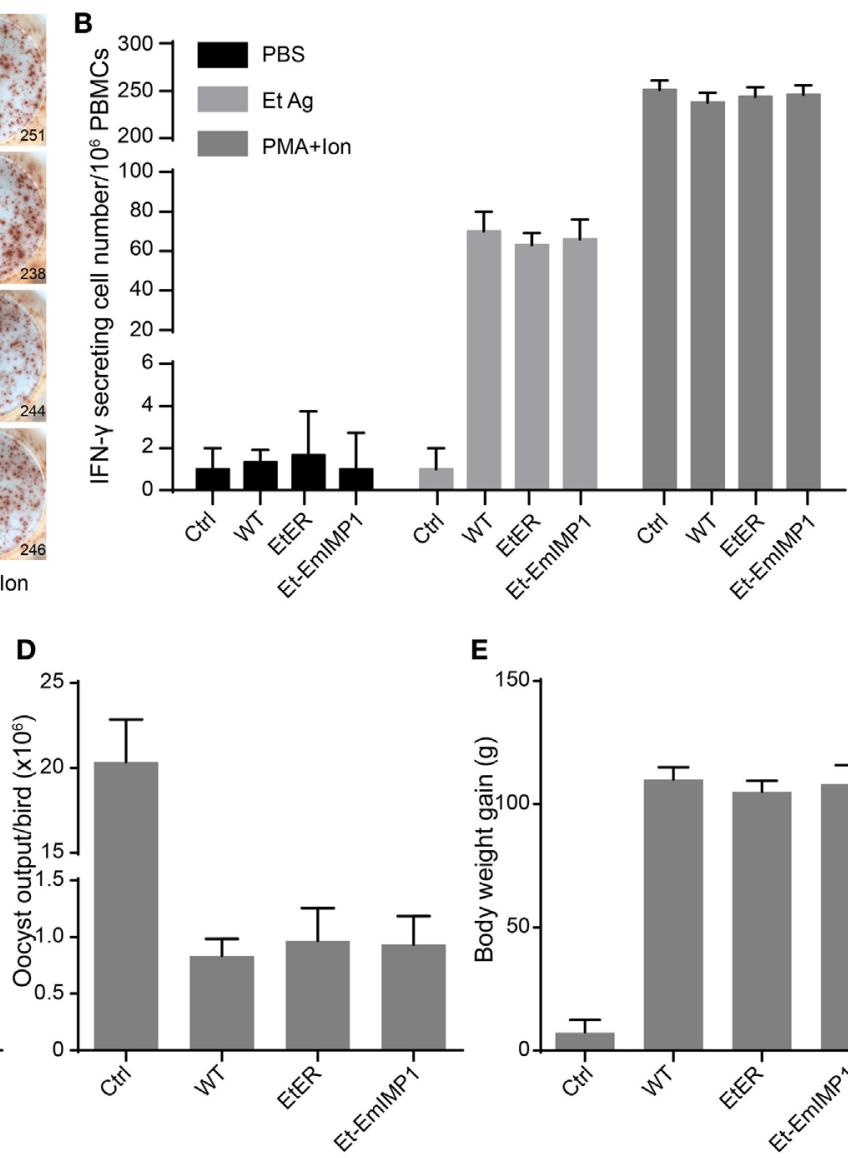

E

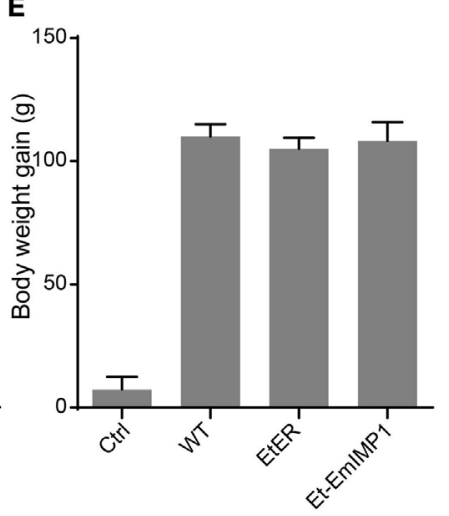

FIGURE 3 | Vaccination with Et-EmIMP1 protected chickens from wild-type Eimeria tenella infection. (A) Et-EmIMP1 with the immunogenicity that stimulated cellular immune responses as its wild type. Peripheral blood mononuclear cells (PBMCs) from naïve (Ctrl), wild-type E. tenella (WT), EtER, and Et-EmIMP1 immunized birds were stimulated with PBS, E. tenella oocyst antigens (Et Ag), and PMA plus ionomycin (PMA + ion, positive control). The number of IFN- $\gamma$ secretion lymphocytes (spots) was measured as described in Section "Materials and Methods." (B) The mean number of Et Ag-specific IFN- $\gamma$ secretion lymphocytes in PBMCs in Et-EmIMP1 and its wild-type immunized birds showed no significant difference $(n=3)$. (C) The Et Ag-specific antibody titer increased after primary $(21$ days) and secondary (35 days) immunization with Et-EmIMP1 as its wild type. (D) Oocyst output after challenge with wild type in the chickens immunized with or without Et-EmIMP1 or its wild type. (E) Body weight gain of chickens after 10 days from challenge infection $(n=6)$. Each value represents the mean $\pm S D$ of six birds. 
the whole parasite antigens of E. maxima were also detected in Et-EmIMP1 immunized chickens, compared to both naïve and WT parasite immunized birds (Figures 4A-C). To test the protective efficacy of these immunities against E. maxima infection, we infected the chickens immunized with or without Et-EmIMP1 or its wild-type parasite with 50 E. maxima oocysts at $35 \mathrm{dpi}$ and analyzed the E. maxima oocyst production. We found significantly reduced oocyst production of Et-EmIMP1 immunized birds' respect to the wild-type and transfection control groups (Figure 4D). Our results also demonstrated the well-established paradigm indicating that there is no cross-protective immunity between different Eimeria species, as the same oocyst output was detected in the naïve and wildtype E. tenella immunized birds after E. maxima infection (Figure 4D).

Floor pen test is an effective intermediate step in the process of translating an anticoccidial vaccine from the laboratory to commercial trials. We vaccinated 1-week-old SPF chickens with 200 Et-EmIMP1 oocysts or its wild type. The chickens were fed in the cages with litter of chopped straw. Half of the chickens of each group were removed to new cages and infected with 50 E. maxima oocysts at 14 and $28 \mathrm{dpi}$, respectively. We found that protective immunity against E. maxima infection was established as early as $14 \mathrm{dpi}$ (Table 1). The immunity was enhanced after self-boosting induced by the offspring oocysts in the litter, as the oocyst production after E. maxima infection of Et-EmIMP1 immunized birds was nearly half of the naive and wild-type parasite immunized birds at $14 \mathrm{dpi}$ and was reduced to nearly $1 / 3$ of the original at $28 \mathrm{dpi}$ (Table $\mathbf{1}$ ).

Immune mapped protein 1 has been identified as immunoprotective antigen from other apicomplexan parasites, such as E. tenella, Toxoplasma, and Neospora (26-28). We tested for the possible cross-protective immunity elicited by the overexpressed EmIMP1 against other Eimeria spp., as it is known that several epitopes are conserved among the seven Eimeria spp. in chickens (Figure S2 in Supplementary Material). We infected the birds with Et-EmIMP1 or wild-type parasite immunization with 50 E. acervulina or E. mitis oocysts and found that at

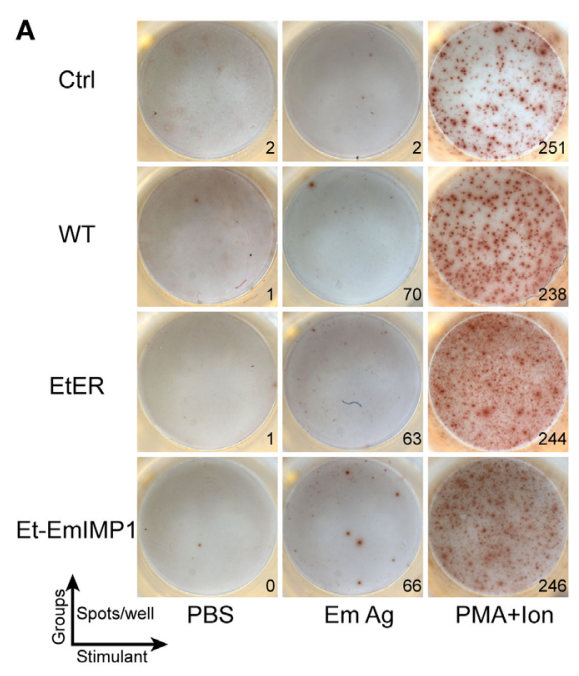

C

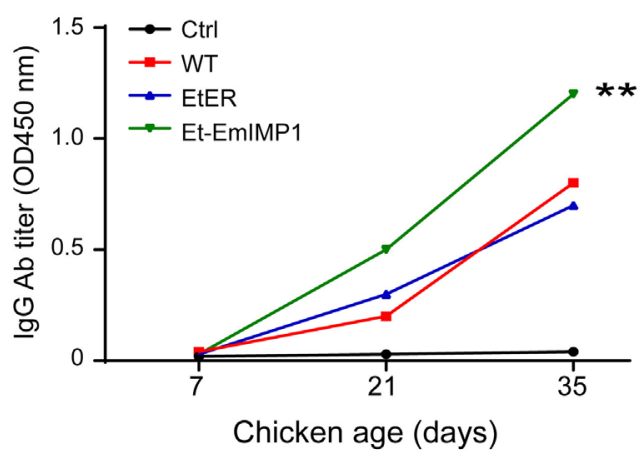

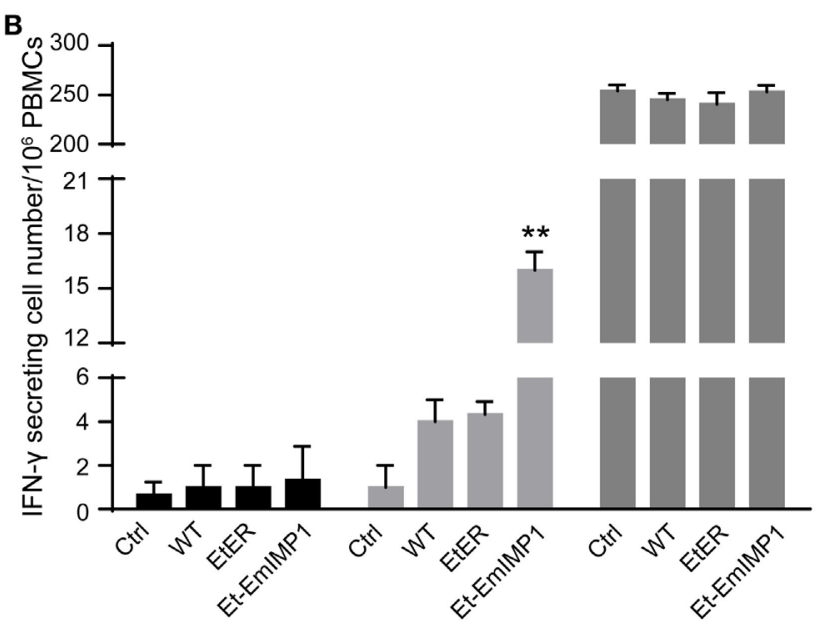

D

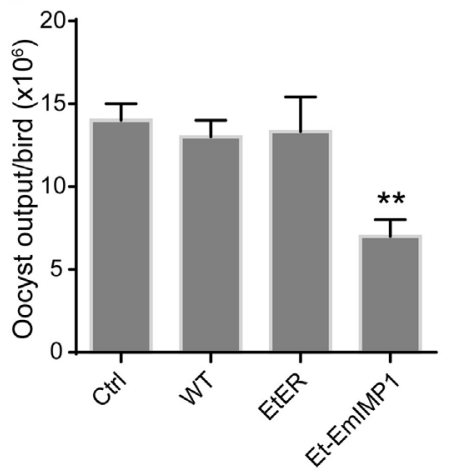

FIGURE 4 | Vaccination with Et-EmIMP1 elicited Eimeria maxima-specific immune responses that partly protected chickens from E. maxima infection. (A) The number of IFN- $\gamma$ secretion lymphocytes (spots) in peripheral blood mononuclear cells (PBMCs) from naïve (Ctrl), wild-type Eimeria tenella (WT), EtER, and Et-EmIMP1 immunized birds after stimulation with PBS, E. maxima oocyst antigens (Em Ag), and PMA plus ionomycin (PMA + ion). (B) Mean number of Em Ag-specific IFN- $\gamma$ secretion lymphocytes in PBMCs in Et-EmIMP1 immunized chickens $(n=3)$. (C) Em Ag-specific antibody titer increased after primary (21 days) and secondary (35 days) immunization with Et-EmIMP1 and was significantly higher than its wild type. (D) Oocyst output after challenge with E. maxima in the chickens immunized with or without Et-EmIMP1 or its wild type $(n=6)$. 
TABLE 1 | Oocyst output following challenge infection with Eimeria maxima in birds vaccinated with or without Et-EmIMP1 or its wild type.

\begin{tabular}{lccc}
\hline Groups & $\begin{array}{c}\text { Immunization } \\
\text { dosage }\end{array}$ & \multicolumn{2}{c}{ Oocyst output/bird $(\times \mathbf{1 0})$} \\
\cline { 3 - 4 } & & $\begin{array}{c}\text { 14 days after } \\
\text { challenge }\end{array}$ & $\begin{array}{c}\mathbf{2 8} \text { days after } \\
\text { challenge }\end{array}$ \\
\hline Ctrl & - & $11.23 \pm 0.35^{\mathrm{a}}$ & $14.37 \pm 0.25^{\mathrm{a}}$ \\
WT & 200 & $12.14 \pm 0.40^{\mathrm{a}}$ & $14.11 \pm 0.30^{\mathrm{a}}$ \\
EtER & 200 & $11.78 \pm 0.30^{\mathrm{a}}$ & $13.73 \pm 0.27^{\mathrm{a}}$ \\
Et-EmIMP1 & 200 & $5.20 \pm 0.27^{\mathrm{b}}$ & $3.60 \pm 0.24^{\mathrm{b}}$ \\
\hline
\end{tabular}

Each value represents the mean $\pm S D$ of three independent immunization/challenge trials. For each column (14 or 28 days), values between groups with different superscripts ( $a$ and $b)$ are significantly different $(p<0.05)$ according to the Duncan's multiple range test.
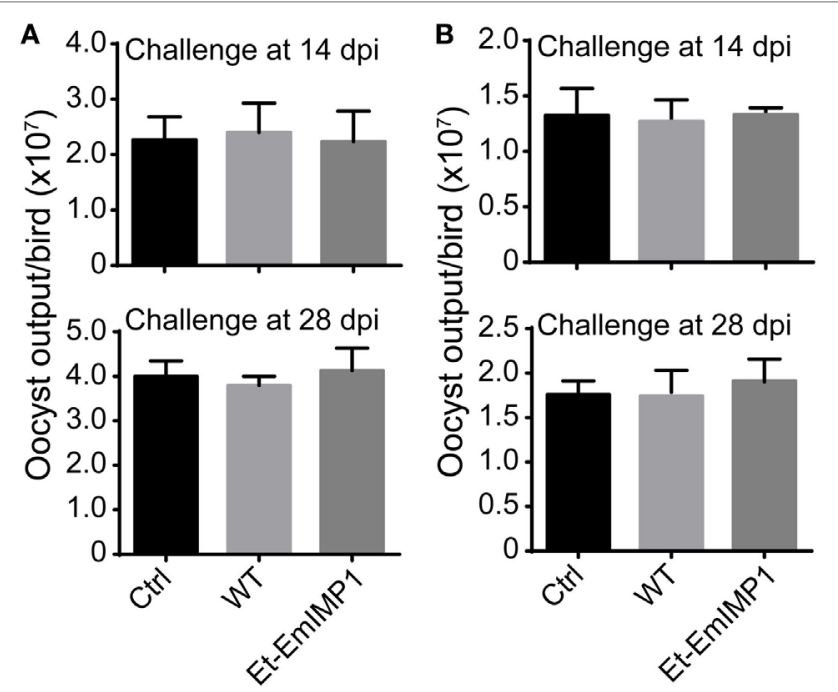

FIGURE 5 | Vaccination with Et-EmIMP1 did not protect chickens from Eimeria acervulina and Eimeria mitis infection. Oocyst output was similar after challenge with $E$. acervulina (A) or E. mitis (B) in the chickens immunized with or without Et-EmIMP1 or its wild type $(n=6)$.

28 dpi there was no significant difference in the E. acervulina (Figure 5A) and E. mitis (Figure 5B) production between immunized and naïve birds. These results demonstrated that protective immunity elicited by EmIMP1 is species faithful and only protected chickens against E. maxima infection but not against the other six species.

\section{DISCUSSION}

In the present study, we demonstrate that transgenic Eimeria parasites used as vaccine delivery model expressing antigen of its affinis species effectively elicited long periods of foreign pathogen-specific protective immune responses, protecting the host against challenges with wild-type paternal and its affinis species. These promising results indicate that transgenic E. tenella expressing immunodominant antigens of other Eimeria parasites, such as E. maxima, is a new and more competitive anticoccidial vaccine strain than traditional ones for the following reasons: (1) the use of strains of E. maxima is not a requirement when immunizing with transgenic E. tenella, thus saving in vaccine production costs $(7,33)$; (2) it eliminates the pathological adverse reaction caused by vaccination with E. maxima; (3) it reduces the "side effects" caused by "selfboosting" immunization because of contact with the offspring oocysts lacking the E. maxima oocysts in the litter (33); and (4) protective immunities are self-boosted during reinfection of the transgenic parasites from the offspring $(6,33)$.

The dose of oocysts intended for each bird varies between vaccine formulations but generally is around 50-100 oocysts for highly immunogenic species such as E. maxima $(5,11)$. The period of establishment of solid immunity also varies between vaccine formulations but generally is around 2-3 weeks for highly immunogenic species such as E. maxima and 3-5 weeks for species such as E. acervulina and E. tenella (10). In contrast, in our study, protective immunity against E. maxima infection was established as early as 14 days after vaccination with Et-EmIMP1 (Table 1). Thus, the immunity provided by the transgenic parasites is sufficient to protect chickens against substantial E. maxima infection because early infection from the environment is equivalent to boosting immunization in transgenic parasite immunized chickens. The time point of solid immunity against E. maxima infection elicited by transgenic Eimeria parasites will be delayed by 1-2 weeks compared to E. maxima. This is still acceptable and satisfactory as middle immunogenic species such as E. acervulina and E. tenella need more than 1 or 2 weeks than E. maxima to build solid immunity $(5,9-11)$.

Live vaccines, based on wild-type parasites, have been available to the poultry industry for around 60 years due to its effective immunities against challenge with homologous parasites. A crucial aspect for the success of this type of vaccines is the method by which they are delivered. Any uneven uptake of vaccinal oocysts by individual birds within a large flock can lead to a series of asynchronous infections during the first 3-5 weeks and even cause coccidiosis outbreaks (5). Live-attenuated vaccines contain the precocious lines of Eimeria that confer a marked attenuation of virulence in comparison to the wild-type strains. The immunogenicity of the precocious line generally remains similar to that of their wild-type parents (34). Immunization with live oocyst formulation vaccines can be achieved by in ovo injection into the embyronating egg (35). This later technique allows the delivery of new types of anticoccidial and other poultry vaccines, but the mechanism by which the oocysts, when injected into the eggs, are able to establish a clear infection in the gut of the developing embryo is not very clear. As with other methods of vaccination, secondary self-boosting immunization from the offspring on the litter is necessary to establish the protective immunity.

Many techniques have been previously used to identify protective antigens of apicomplexan parasites with the objective of developing subunit vaccines, including coccidiosis vaccines. Immunizing hens with affinity-purified antigens from the wall forming bodies of macrogametocytes of E. maxima is the only technique with a subunit vaccine currently employed against any protozoan parasite $(36,37)$. Maternal antibodies pass via the egg to the newly hatched chick and provide passive protection of limited duration $(5,6,10)$. Immunizing chicks with the 
recombinant antigens of eimerian parasites, including IMP1, AMA1, profilin, etc., produced by Escherichia coli or DNA vaccines also provides partial protection $(1,12,38,39)$. To improve the immunogenicity of the subunit vaccines, various types of molecular adjuvants, such as toll-like receptor ligand or co-stimulators of $\mathrm{T}$ cells and cytokines, were introduced by fusing expression with the antigens or co-immunizing with them (40-42). Although enhanced immune responses were detected after immunization that was two to three times higher using subunit vaccines together with the adjuvants, they can hardly protect chickens from subsequent infections of Eimeria parasites $(22,43)$. For this reason, the vaccine delivery vehicle in which Eimeria parasites release the antigens as the parasites infect the hosts constitute a breakthrough technique, but it also provides a new direction for the development of recombinant vaccines in livestock and possibly even in humans.

Immunity to Eimeria is complex and multifactorial. Anti-CD4 depletion studies in chickens demonstrated that CD4+ but not CD8+ cells were essential for resistance to primary infection with E. tenella but not E. acervulina (44). The major mediator of immune-mediated resistance to primary infection and parasites killing is IFN- $\gamma$, although the source of effective IFN- $\gamma$ differs between different Eimeria species $(45,46)$. Studies with B celldeficient mice (47) and bursectomised chickens (48) suggest that B cells play a minor role during Eimeria parasite primary infection and are not essential for expression of complete immunity to re-challenge infection, although specific immunoglobulin (IgM, IgG, and IgA) is induced by infection.

In conclusion, the transgenic Eimeria parasite expressing immunodominant antigens of other Eimeria species provides a new strategy for the development of coccidiosis vaccines. We are convinced that our strategy can be extended to the development of E. tenella as a vector carrying the immunodominant antigens of Eimeria necatrix, reducing the need for formulations of E. necatrix or other pathogenic Eimeria species (49). The most promising coccidiosis vaccines will be the ones containing only one species of transgenic Eimeria while providing protective immunity against all the Eimeria parasites infections. This proposed strategy relies on the use of multiple techniques, including considerable use of molecular biology techniques to identify antigens capable of inducing an immune response. Our results have important implications for the development of live sporozoite malaria vaccines in which antigens from different strains can be expressed in the vaccine strain.

\section{REFERENCES}

1. Blake DP, Clark EL, Macdonald SE, Thenmozhi V, Kundu K, Garg R, et al. Population, genetic, and antigenic diversity of the apicomplexan Eimeria tenella and their relevance to vaccine development. Proc Natl Acad Sci U S A (2015) 112:E5343-50. doi:10.1073/pnas.1506468112

2. Chang Z, Jiang N, Zhang Y, Lu H, Yin J, Wahlgren M, et al. The TatD-like DNase of Plasmodium is a virulence factor and a potential malaria vaccine candidate. Nat Commun (2016) 7:11537. doi:10.1038/ncomms11537

3. Ma M, Baumgartner M. Intracellular Theileria annulata promote invasive cell motility through kinase regulation of the host actin cytoskeleton. PLoS Pathog (2014) 10:e1004003. doi:10.1371/journal.ppat.1004003

\section{ETHICS STATEMENT}

All animal experiments were performed in strict accordance with the China Agricultural University Institutional Animal Care and Use Committee guidelines (CAU20160628-2) and followed the International Guiding Principles for Biomedical Research Involving Animals. Experiments were approved by the Beijing Administration Committee of Laboratory Animals.

\section{AUTHOR CONTRIBUTIONS}

$\mathrm{XT}$ and XS conceived and designed this study and analyzed the data. XT carried out the experiments and drafted the manuscripts. XL, GY, JS, GT, and SZ contributed to help the statistical analysis and help to draft the manuscripts. XS and XL supervised the study implementation and revised the manuscript. All authors read and approved the final version of the manuscript.

\section{ACKNOWLEDGMENTS}

This study was supported by the National Natural Science Foundation of China (31330076 and 31572507). We are most grateful to Dr. Antonio Peramo for comments and revisions that greatly improved this manuscript. We also thank Chao $\mathrm{Li}$, Chaoyue Wang, and Xiuling Tian in our laboratory for their generous assistance during the study.

\section{SUPPLEMENTARY MATERIAL}

The Supplementary Material for this article can be found online at https://www.frontiersin.org/articles/10.3389/fimmu.2017.01982/ full\#supplementary-material.

FIGURE S1 | Vaccination with Et-EmIMP1 elicited EmIMP1-specific immune responses. (A) Mean number of EmIMP1-specific IFN- $\gamma$ secretion lymphocytes in peripheral blood mononuclear cells (PBMCs) in Et-EmIMP1 immunized chickens $(n=3)$. (B) EmIMP1-specific antibody titer increased after primary (21 days) and secondary (35 days) immunization with Et-EmIMP1 significantly higher than its wild type. Each value represents the mean \pm SD of three birds. ${ }^{*} p<0.05$; ${ }^{* *} p<0.01$.

FIGURE S2 | Multiple alignment of immune mapped protein 1 (IMP1) of chicken coccidia. Immune mapped protein 1 of E. maxima (EmIMP1)'s gene number is given in the Section "Materials and Methods." GeneDB's gene numbers of IMP1 of Eimeria tenella, Eimeria acervulina, Eimeria necatrix, Eimeria mitis, Eimeria brunette, and Eimeria praecox were ETH_00030475, EAH_00011210, ENH_00036180, EMH_0033960, EBH_0052010, and EPH_0045280, respectively.

4. Montoya JG, Liesenfeld O. Toxoplasmosis. Lancet (2004) 363:1965-76 doi:10.1016/S0140-6736(04)16412-X

5. Shirley MW, Smith AL, Tomley FM. The biology of avian Eimeria with an emphasis on their control by vaccination. Adv Parasitol (2005) 60:285-330. doi:10.1016/S0065-308X(05)60005-X

6. Chapman HD, Cherry TE, Danforth HD, Richards G, Shirley MW, Williams RB. Sustainable coccidiosis control in poultry production: the role of live vaccines. Int J Parasitol (2002) 32:617-29. doi:10.1016/S0020-7519(01) 00362-9

7. Blake DP, Tomley FM. Securing poultry production from the ever-present Eimeria challenge. Trends Parasitol (2014) 30:12-9. doi:10.1016/ j.pt.2013.10.003 
8. Suo X, Zhang JX, Li ZG, Yang CT, Min QR, Xu LT, et al. The efficacy and economic benefits of Supercox, a live anticoccidial vaccine in a commercial trial in broiler chickens in China. Vet Parasitol (2006) 142:63-70. doi:10.1016/ j.vetpar.2006.06.020

9. Williams RB. Fifty years of anticoccidial vaccines for poultry (19522002). Avian Dis (2002) 46:775-802. doi:10.1637/0005-2086(2002)046 [0775:FYOAVF]2.0.CO;2

10. Chapman HD, Barta JR, Blake D, Gruber A, Jenkins M, Smith NC, et al. A selective review of advances in coccidiosis research. Adv Parasitol (2013) 83:93-171. doi:10.1016/B978-0-12-407705-8.00002-1

11. Shirley MW, Lillehoj HS. The long view: a selective review of 40 years of coccidiosis research. Avian Pathol (2012) 41:111-21. doi:10.1080/03079457 .2012 .666338

12. Blake DP, Billington KJ, Copestake SL, Oakes RD, Quail MA, Wan KL, et al. Genetic mapping identifies novel highly protective antigens for an apicomplexan parasite. PLoS Pathog (2011) 7:e1001279. doi:10.1371/journal. ppat.1001279

13. Clark JD, Oakes RD, Redhead K, Crouch CF, Francis MJ, Tomley FM, et al. Eimeria species parasites as novel vaccine delivery vectors: anti-Campylobacter jejuni protective immunity induced by Eimeria tenella-delivered CjaA. Vaccine (2012) 30:2683-8. doi:10.1016/j.vaccine.2012.02.002

14. Marugan-Hernandez V, Cockle C, Macdonald S, Pegg E, Crouch C, Blake DP, et al. Viral proteins expressed in the protozoan parasite Eimeria tenella are detected by the chicken immune system. Parasit Vectors (2016) 9:463. doi:10.1186/s13071-016-1756-2

15. Tang X, Yin G, Qin M, Tao G, Suo J, Liu X, et al. Transgenic Eimeria tenella as a vaccine vehicle: expressing TgSAG1 elicits protective immunity against Toxoplasma gondii infections in chickens and mice. Sci Rep (2016) 6:29379. doi:10.1038/srep29379

16. Long PL, Millard BJ, Joyner LP, Norton CC. A guide to laboratory techniques used in the study and diagnosis of avian coccidiosis. Folia Vet Lat (1976) 6:201-17.

17. Tang X, Liu X, Tao G, Qin M, Yin G, Suo J, et al. "Self-cleaving” 2A peptide from porcine teschovirus-1 mediates cleavage of dual fluorescent proteins in transgenic Eimeria tenella. Vet Res (2016) 47:68. doi:10.1186/s13567-016-0351-Z

18. Liu X, Shi T, Ren H, Su H, Yan W, Suo X. Restriction enzyme-mediated transfection improved transfection efficiency in vitro in Apicomplexan parasite Eimeria tenella. Mol Biochem Parasitol (2008) 161:72-5. doi:10.1016/ j.molbiopara.2008.06.006

19. Yin G, Liu X, Zou J, Huang X, Suo X. Co-expression of reporter genes in the widespread pathogen Eimeria tenella using a double-cassette expression vector strategy. Int J Parasitol (2011) 41:813-6. doi:10.1016/j.ijpara.2011.04.001

20. Haug A, Williams RB, Larsen S. Counting coccidial oocysts in chicken faeces: a comparative study of a standard McMaster technique and a new rapid method. Vet Parasitol (2006) 136:233-42. doi:10.1016/j.vetpar.2005.11.024

21. Li Z, Tang X, Suo J, Qin M, Yin G, Liu X, et al. Transgenic Eimeria mitis expressing chicken interleukin 2 stimulated higher cellular immune response in chickens compared with the wild-type parasites. Front Microbiol (2015) 6:533. doi:10.3389/fmicb.2015.00533

22. Yin G, Qin M, Liu X, Suo J, Suo X. Interferon-gamma enzyme-linked immunosorbent spot assay as a tool to study $\mathrm{T}$ cell responses to Eimeria tenella infection in chickens. Poult Sci (2013) 92:1758-63. doi:10.3382/ps.2012-02998

23. Hanig S, Entzeroth R, Kurth M. Chimeric fluorescent reporter as a tool for generation of transgenic Eimeria (Apicomplexa, Coccidia) strains with stage specific reporter gene expression. Parasitol Int (2012) 61:391-8. doi:10.1016/ j.parint.2012.01.010

24. Walker RA, Niepceron A, Ramakrishnan C, Sedano L, Hehl AB, Brossier F, et al. Discovery of a tyrosine-rich sporocyst wall protein in Eimeria tenella. Parasit Vectors (2016) 9:124. doi:10.1186/s13071-016-1410-Z

25. Yan W, Liu X, Shi T, Hao L, Tomley FM, Suo X. Stable transfection of Eimeria tenella: Constitutive expression of the YFP-YFP molecule throughout the life cycle. Int J Parasitol (2009) 39:109-17. doi:10.1016/j.ijpara.2008.06.013

26. Cui X, Lei T, Yang DY, Hao P, Liu Q. Identification and characterization of a novel Neospora caninum immune mapped protein 1. Parasitology (2012) 139:998-1004. doi:10.1017/S0031182012000285

27. Cui X, Lei T, Yang D, Hao P, Li B, Liu Q. Toxoplasma gondii immune mapped protein-1 (TgIMP1) is a novel vaccine candidate against toxoplasmosis. Vaccine (2012) 30:2282-7. doi:10.1016/j.vaccine.2012.01.073

28. Yin G, Qin M, Liu X, Suo J, Tang X, Tao G, et al. An Eimeria vaccine candidate based on Eimeria tenella immune mapped protein 1 and the TLR-5 agonist
Salmonella typhimurium FliC flagellin. Biochem Bioph Res Commun (2013) 440:437-42. doi:10.1016/j.bbrc.2013.09.088

29. Pogonka T, Schelzke K, Stange J, Papadakis K, Steinfelder S, Liesenfeld $\mathrm{O}$, et al. CD8+ cells protect mice against reinfection with the intestinal parasite Eimeria falciformis. Microbes Infect (2010) 12:218-26. doi:10.1016/ j.micinf.2009.12.005

30. Huang X, Zou J, Xu H, Ding Y, Yin G, Liu X, et al. Transgenic Eimeria tenella expressing enhanced yellow fluorescent protein targeted to different cellular compartments stimulated dichotomic immune responses in chickens. J Immunol (2011) 187:3595-602. doi:10.4049/jimmunol.1100043

31. Rothwell L, Muir W, Kaiser P. Interferon-gamma is expressed in both gut and spleen during Eimeria tenella infection. Avian Pathol (2000) 29:333-42. doi:10.1080/03079450050118467

32. Smith NC, Wallach M, Petracca M, Braun R, Eckert J. Maternal transfer of antibodies induced by infection with Eimeria maxima partially protects chickens against challenge with Eimeria tenella. Parasitology (1994) 109(Pt 5):551-7. doi:10.1017/S0031182000076423

33. Williams RB. Quantification of the crowding effect during infections with the seven Eimeria species of the domesticated fowl: its importance for experimental designs and the production of oocyst stocks. Int J Parasitol (2001) 31:1056-69. doi:10.1016/S0020-7519(01)00235-1

34. Shirley MW. Development of a live attenuated vaccine against coccidiosis of poultry. Parasite Immunol (1989) 11:117-24. doi:10.1111/j.1365-3024.1989. tb00653.x

35. Weber FH, Genteman KC, LeMay MA, Lewis DS, Evans NA. Immunization of broiler chicks by in ovo injection of infective stages of Eimeria. Poult Sci (2004) 83:392-9. doi:10.1093/ps/83.3.392

36. Sharman PA, Smith NC, Wallach MG, Katrib M. Chasing the golden egg: vaccination against poultry coccidiosis. Parasite Immunol (2010) 32:590-8. doi:10.1111/j.1365-3024.2010.01209.x

37. Wallach MG, Ashash U, Michael A, Smith NC. Field application of a subunit vaccine against an enteric protozoan disease. PLoS One (2008) 3:e3948. doi:10.1371/journal.pone.0003948

38. Song KD, Lillehoj HS, Choi KD, Yun CH, Parcells MS, Huynh JT, et al. A DNA vaccine encoding a conserved Eimeria protein induces protective immunity against live Eimeria acervulina challenge. Vaccine (2000) 19:243-52. doi:10.1016/S0264-410X(00)00169-9

39. Song X, Gao Y, Xu L, Yan R, Li X. Partial protection against four species of chicken coccidia induced by multivalent subunit vaccine. Vet Parasitol (2015) 212:80-5. doi:10.1016/j.vetpar.2015.08.026

40. Agrawal S, Agrawal A, Doughty B, Gerwitz A, Blenis J, Van Dyke T, et al. Cutting edge: different Toll-like receptor agonists instruct dendritic cells to induce distinct Th responses via differential modulation of extracellular signal-regulated kinase-mitogen-activated protein kinase and c-Fos. J Immunol (2003) 171:4984-9. doi:10.4049/jimmunol.171.10.4984

41. Hashem AM, Gravel C, Chen Z, Yi Y, Tocchi M, Jaentschke B, et al. CD40 ligand preferentially modulates immune response and enhances protection against influenza virus. J Immunol (2014) 193:722-34. doi:10.4049/jimmunol.1300093

42. Rochman Y, Spolski R, Leonard WJ. New insights into the regulation of T cells by gamma(c) family cytokines. Nat Rev Immunol (2009) 9:480-90. doi:10.1038/nri2580

43. Qin M, Tang X, Yin G, Liu X, Suo J, Tao G, et al. Chicken IgY Fc expressed by Eimeria mitis enhances the immunogenicity of E. mitis. Parasit Vectors (2016) 9:164. doi:10.1186/s13071-016-1451-3

44. Trout JM, Lillehoj HS. T lymphocyte roles during Eimeria acervulina and Eimeria tenella infections. Vet Immunol Immunopathol (1996) 53:163-72. doi:10.1016/0165-2427(95)05544-4

45. Rothwell L, Young JR, Zoorob R, Whittaker CA, Hesketh P, Archer A, et al. Cloning and characterization of chicken IL-10 and its role in the immune response to Eimeria maxima. J Immunol (2004) 173:2675-82. doi:10.4049/ jimmunol.173.4.2675

46. Smith AL, Hayday AC. Genetic dissection of primary and secondary responses to a widespread natural pathogen of the gut, Eimeria vermiformis. Infect Immun (2000) 68:6273-80. doi:10.1128/IAI.68.11.6273-6280.2000

47. Smith AL, Hayday AC. Genetic analysis of the essential components of the immunoprotective response to infection with Eimeria vermiformis. Int J Parasitol (1998) 28:1061-9. doi:10.1016/S0020-7519(98)00081-2

48. Rose ME, Hesketh P. Immunity to coccidiosis: T-lymphocyte- or B-lymphocyte-deficient animals. Infect Immun (1979) 26:630-7. 
49. Montes C, Rojo F, Hidalgo R, Ferre I, Badiola C. Selection and development of a Spanish precocious strain of Eimeria necatrix. Vet Parasitol (1998) 78:169-83. doi:10.1016/S0304-4017(98)00125-3

Conflict of Interest Statement: The authors declare that the research was conducted in the absence of any commercial or financial relationships that could be construed as a potential conflict of interest.
Copyright (c) 2018 Tang, Liu, Yin, Suo, Tao, Zhang and Suo. This is an open-access article distributed under the terms of the Creative Commons Attribution License (CC BY). The use, distribution or reproduction in other forums is permitted, provided the original author(s) or licensor are credited and that the original publication in this journal is cited, in accordance with accepted academic practice. No use, distribution or reproduction is permitted which does not comply with these terms. 